\title{
Application of in vitro CYP and transporter assays to predict clinical drug-drug interactions
}

\author{
Donna A Volpe*,1 \& Praveen V Balimane ${ }^{1}$ \\ ${ }^{1}$ Office of Clinical Pharmacology, Center for Drug Evaluation \& Research, Food \& Drug Administration, Silver Spring, MD 20933, \\ USA \\ *Author for correspondence: Donna.Volpe@fda.hhs.gov
}

First draft submitted: 4 December 2017; Accepted for publication: 22 February 2018; Published online: 16 May 2018

Keywords: drug interactions $\bullet$ drug-metabolizing enzymes $\bullet$ drug transporters $\bullet$ mass spectrometry

Drug transporters and drug-metabolizing enzymes participate in the processes of absorption, disposition, metabolism and elimination, which influence the pharmacokinetics and pharmacodynamics of a new chemical entity (NCE). Therefore, understanding an NCE's potential as a substrate, inhibitor or inducer of a drug-metabolizing enzyme or transporter has become an essential element of risk assessment during drug discovery, development and regulatory review [1-7].

\section{Drug metabolism}

Drugs are metabolized by Phase I and/or II enzymes in several organs, especially the liver, intestine and kidneys. The aim of drug metabolism is to increase the polarity and solubility of the molecule to enable its elimination. Phase I metabolic reactions introduce reactive and polar groups into the substrate NCE. Cytochrome P450 (CYP) enzymes integrate an oxygen atom into nonactivated hydrocarbons, resulting in either the introduction of hydroxyl groups or the $N-, O$ - and $S$-dealkylation of substrates. Other Phase I enzymes include the flavin monooxygenases, monoamine oxidases and xanthine oxidase/aldehyde oxidase. Outcomes of Phase I metabolism include inactivation of the drug by production of pharmacologically inactive metabolites or generation of active metabolite(s). Phase II metabolic reactions include glucuronidation, sulfonation, methylation, acetylation and glutathione conjugation. Phase II enzymes include uridine-5'-diphospho-glucuronosyltransferases, sulfotransferases, glutathione $S$-transferases and $N$-acetyltransferases. Metabolites formed during Phase II reactions are not likely to be pharmacologically active.

\section{Drug transport}

Uptake and efflux transporters determine plasma and tissue concentrations of both endogenous and exogenous substances. The transporters are found in the small intestines, liver and kidneys, which are critical for drug absorption and elimination. They are also located in vital blood-tissue barriers such as the blood-brain and blood-placenta barriers. Solute carrier transporters facilitate the uptake of compounds into cells through facilitated diffusion or via cotransport with intracellular and/or extracellular ions. ATP-binding cassette transporters efflux compounds out of cells against a concentration gradient driven by the hydrolysis of ATP as an energy source.

\section{Drug interactions}

Drug-drug interactions occur when one drug ('perpetrator') interacts with another drug ('victim') by altering its metabolism and/or transport following coadministration. Drug-drug interactions can have serious clinical effects by decreasing the therapeutic efficacy or increasing the toxicity of a victim drug. Polypharmacy, the simultaneous use of multiple drugs in a patient for one or more conditions, increases the risk for drug interactions. Evaluation 
of drug transporters and enzymes in the discovery and development may lead to a better understanding of how they affect a new drug's oral bioavailability, systemic exposure, toxicity, efficacy, tissue distribution and drug-drug interactions $[8,9]$.

\section{In vitro assays}

During drug discovery and development phases, in vitro assays are important tools to identify substrates, inducers and inhibitors of transporters and drug-metabolizing enzymes. Regulatory guidances offer an outline for evaluating metabolism- and transporter-based interactions to establish the need for clinical interaction studies and inform drug product labeling $[6-7,10]$. Drug interaction evaluations are designed to identify the principal route of a drug's elimination, estimate the contribution of transporters and drug-metabolizing enzymes to a drug's disposition, and characterize a drug's effect(s) on transporters and drug-metabolizing enzymes [6]. The in vitro assays assess the potential for clinically significant drug interactions by determining whether the NCE may alter the pharmacokinetics of another drug, if other drugs can alter the pharmacokinetics of the NCE, and/or the extent of these modifications on a victim drug's pharmacokinetic parameters.

Based on the results of the in vitro assays to determine whether an NCE is a substrate or inhibition of drugmetabolizing enzymes or transporter, a clinical study may be needed to further delineate drug interaction(s) with the NCE [7,10]. The results of the clinical interaction studies are communicated in the drug product label [10].

For CYP-based interactions, the initial step is to ascertain the CYP isoform(s) responsible for the metabolism of the NCE. These reaction-phenotyping experiments identify which enzymes, and their proportion to overall metabolism, for the NCE and its potential 'victim' in drug interactions [5]. There are complementary methods using human liver microsomes (HLM), recombinant (r) CYP enzymes, S9 fractions and human hepatocytes in conjunction with isoform-specific chemical inhibitors or inhibitory antibodies to examine a drug's enzymemediated elimination pathways. These reaction-phenotyping experiments measure substrate depletion and/or metabolite generation.

CYP inhibition assays similarly utilize HLM, rCYP and human hepatocytes to analyze reduction of metabolism with determination of $\mathrm{IC}_{50}$ (concentration at $50 \%$ inhibition), $\mathrm{K}_{\mathrm{i}}$ (equilibrium dissociation constant), $\mathrm{k}_{\text {inact }}$ (maximum rate of inactivation) and $\mathrm{K}_{\mathrm{I}}$ (inhibitor concentration at half-maximal inactivation rate) values [11]. Probe substrates used in the inhibition experiments should be selective (i.e., predominantly metabolized by a single CYP enzyme) and have simple a metabolic scheme (i.e., does not undergo sequential metabolism) [6]. Reversible inhibition encompasses the rapid association and dissociation of drugs and their enzymes and the interactions are often classified as competitive, noncompetitive or uncompetitive. In contrast, time-dependent inhibition (TDI) interactions display a delayed onset as a result of the time dependence in their inhibitory effects. These effects can persist even after the inhibitor has been eliminated since enzymatic activity is only restored by new protein synthesis of the affected enzyme.

For reversible inhibition assays, the reaction mixture includes the enzyme source (e.g., HLM, rCYP), a probe substrate at its $\mathrm{K}_{\mathrm{m}}$ (concentration at half-maximal enzyme velocity), a NADPH-regenerating system (NRS) and the NCE or control inhibitor at multiple concentrations [12]. After the reaction is stopped, the metabolite is measured followed by calculation of $\mathrm{IC}_{50}$ and/or $\mathrm{K}_{\mathrm{i}}$ values. To increase the throughput in enzyme inhibition experiments, several substrates may be included in the reaction mixture to assess multiple CYP activities simultaneously within a single experiment (the cocktail approach) $[13,14]$. This approach takes advantage of the selectivity and specificity of LC-MS analysis to accurately monitor the various CYP activities in the experiment.

An $\mathrm{IC}_{50}$ shift assay is first used as an indicator for TDI potential at multiple concentrations of the NCE [15]. For these experiments, the NCE are preincubated with HLM or rCYP in the presence or absence of the NRS. At the end of the incubation, aliquots of the preincubation mixture are diluted into secondary incubations containing the probe CYP substrate at its $\mathrm{K}_{\mathrm{m}}$ concentration and NRS. The resultant data are used to determine $\mathrm{IC}_{50}$ values with and without NRS. Compounds that exhibit an $\mathrm{IC}_{50}$ shift undergo further analysis for TDI. HLM or rCYP are preincubated with the NCE and NRS and at multiple time points, aliquots are removed from the preincubation mixture and diluted into secondary incubations containing the probe substrate at five-times its $\mathrm{K}_{\mathrm{m}}$ concentration, and NRS. Results from these experiments are used to calculate $K_{I}$ and $k_{\text {inact }}$ values for the NCE [16].

Primary human hepatocytes are the most acceptable assay system to assess the extent of induction of CYP enzymes by an NCE [3,6-7]. Induction of CYPs usually occurs through activation of nuclear receptors that regulate gene transcription. The standard method for measuring enzyme induction is to incubate the NCE with plated 
human hepatocytes for 2-3 days. Following incubation, changes in mRNA expression and/or CYP enzyme activity with a probe substrate are quantified with comparison to a known inducer.

Drug-transporter assays can be classified as membrane- or cell-based [1,4]. The membrane-based assays take advantage of the location of efflux transporters in the membrane for drug interaction. The membranes are prepared from cells expressing the transporter to identify efflux substrates and inhibitors. The cell-based systems assess the passage of drugs across cell membranes expressing efflux or uptake transporters. Given their intact cell structures, these assays may impart more complete information about the interaction between the tested drugs and transporters [4].

Inside-out vesicles are generated from plasma membranes isolated from transporter-expressing cells, so that substrates are effluxed into the vesicle in the presence of ATP. Employing a rapid filtration method, the membrane vesicles are maintained on a filter membrane and the compounds collected in the vesicles are then measured. Effluxtransporter inhibitors may be characterized via the intracellular retention of a probe substrate in the presence of a test compound. In accumulation assays, the ability to inhibit the efflux transporter is evaluated by the accumulation of a probe substrate marker in cells expressing high levels of the transporter relative to wild-type or nontransfected cells. Enhanced accumulation of a probe substrate in the presence of the NCE demonstrates inhibition of the efflux transporter.

Bidirectional assays are the most direct and accepted models for determining whether an NCE is a substrate or inhibitor of an efflux transporter [1,4]. Cell lines (e.g., Caco-2, MDCK) or transfected cells are cultured in a dual chamber apparatus to form confluent and polarized monolayers on a semiporous filter that separates the apical and basolateral chambers. An efflux ratio is calculated comparing permeability of the NCE or probe substrate in basolateral-to-apical to apical-to-basolateral directions. An NCE is deemed to be an inhibitor if it decreases the efflux ratio of a probe substrate across the cell monolayer.

Uptake assays for solute carrier transporters may be performed with suspended or plated transfected cells or hepatocytes. To determine if an NCE is a transporter substrate, it is incubated with the cells for a brief time period followed by measurement of the drug's intracellular concentration. This concentration is then normalized to cell number or protein content of the cell lysate. Alternatively, an NCE is considered to be an inhibitor if it decreases the uptake of a probe substrate into the cells.

It is important to optimize and validate the in vitro transporter and metabolism assays to predict an NCE's interaction potential. This is accomplished by: use of negative and positive control substrates and inhibitors, understanding of how experimental variability affects assay outcome, and establishing acceptance criteria for experimental results with the negative and positive controls [4]. This also includes the analytical assays utilized to measure the NCE, probe substrate or metabolite resulting from the transporter and metabolism experiments. An important aspect of using LC-MS methods in transporter and metabolism assays is that they be reliable and reproducible for their intended use. This is achieved via validation of the analytical procedures by demonstrating: selectivity; accuracy, precision and recovery; linearity (calibration curve); sensitivity; reproducibility; and analyte stability [17].

\section{Analytical assays}

MS assays are generally used to measure substrates and metabolites in drug transporter and metabolism in vitro assays $[1,11,18]$. MS assays provide selectivity and sensitivity for analyte detection and are readily automated for the experiments to improve throughput.

LC-MS/MS is a valuable tool for early metabolism investigations, when information of an NCE's major metabolites are limited making chromatographic separation impractical. Through monitoring of commonly expected biotransformation products of the CYP enzymes (e.g., monooxygenation, dealkylation, carboxylation, etc.) of parent mass and possible fragmentation products provides an initial indication of potential metabolic pathways of an NCE. While LC-MS remains the standard for analytical methodology, its throughput is limited by the long elution time in the chromatography phase [19]. A newer technique, RapidFire ${ }^{T M}$ mass spectroscopy, combines microscale solid-phase extraction with rapid column switching that is directly coupled to a triple-quadrupole mass spectrometer. RapidFire ${ }^{\top M}$ MS is fully automated and completes integrated sample purification and MS analysis with reduced sample cycle times [2,19]. In reaction-phenotyping experiments, high-resolution mass spectrometry is able to follow the disappearance of the parent compound and concurrently identify and monitor the formation of metabolites in each enzymatic system being evaluated for the NCE [20]. 


\section{Conclusion}

Understanding transporter and metabolism drug-drug interactions is essential to the risk/benefit assessment of an NCE. The objectives of in vitro transporter and metabolism assays in drug discovery and development are to identify an NCE as a substrate and/or inhibitor of metabolic enzymes and/or transporters, model and predict potential drug interactions, design essential clinical interaction studies and inform drug product labels $[4,6,10]$. The optimal utilization of these in vitro drug interaction assays is a powerful tool in the research and development continuum and can contribute in both the drug discovery stage (i.e., selection of the right compound) as well as the development stage (i.e., devising the right strategy for assessing and managing the drug interaction effects).

\section{Disclaimer}

The findings and conclusions in this article have not been formally disseminated by the US FDA and should not be construed to represent any Agency endorsement, determination or policy.

\section{Financial \& competing interests disclosure}

The authors have no relevant affiliations or financial involvement with any organization or entity with a financial interest in or financial conflict with the subject matter or materials discussed in the manuscript. This includes employment, consultancies, honoraria, stock ownership or options, expert testimony, grants or patents received or pending, or royalties.

No writing assistance was utilized in the production of this manuscript.

\section{References}

1. Brouwer KL, Keppler D, Hoffmaster KA et al. In vitro methods to support transporter evaluation in drug discovery and development. Clin. Pharmacol. Ther. 94(1), 95-112 (2013).

2. Foti RS, Wienkers LC, Wahlstrom JL. Application of cytochrome P450 drug interaction screening in drug discovery. Comb. Chem. High Throughput Screen. 13(2), 145-158 (2010).

3. Honma M, Kozawa M, Suzuki H. Methods for the quantitative evaluation and prediction of CYP enzyme induction using human in vitro systems. Expert Opin. Drug Discov. 5(5), 491-511 (2010).

4. Volpe DA. Transport assays as useful in vitro tools in drug development. Expert Opin. Drug Discov. 11(1), 91-103 (2016).

5. Zientek MA, Youdim K. Reaction phenotyping: advances in the experimental strategies used to characterize the contribution of drug-metabolizing enzymes. Drug Metab. Dispos. 43(1), 163-181 (2015).

6. US FDA. Guidance for Industry. In Vitro Metabolism- and Transporter-Mediated Drug-Drug Interaction Studies(2017). www.fda.gov/downloads/drugs/guidances/ucm292362.pdf

7. European Medicines Agency. Guideline on the Investigation of Drug Interactions(2012). www.ema.europa.eu/docs/en_GB/document_library/Scientific_guideline/2012/07/WC500129606.pdf

8. Kim RB. Transporters and drug discovery: why, when, and how. Mol. Pharm. 3(1), 26-32 (2006).

9. Zhang L, Zhang YD, Zhao P, Huang SM. Predicting drug-drug interactions: an FDA perspective. AAPS J. 11(2), 300-306 (2009).

10. US FDA. Guidance for Industry. Clinical Drug Interaction Studies - Study Design, Data Analysis, and Clinical Implications (2017). www.fda.gov/downloads/drugs/guidances/ucm292362.pdf

11. Fowler S, Zhang H. In vitro evaluation of reversible and irreversible cytochrome P450 inhibition: current status on methodologies and their utility for predicting drug-drug interactions. AAPS J. 10(2), 410-424 (2008).

12. Walsky RL, Obach RS. Validated assays for human cytochrome P450 activities. Drug Metab. Dispos. 32(6), 647-660 (2004).

13. Spaggiari D, Geiser L, Daali Y, Rudaz S. A cocktail approach for assessing the in vitro activity of human cytochrome P450s: an overview of current methodologies. J. Pharm. Biomed. Anal. 101, 221-237 (2014).

14. Zambon S, Fontana S, Kajbaf M. Evaluation of cytochrome P450 inhibition assays using human liver microsomes by a cassette analysis /LC-MS/MS. Drug Metab. Lett. 4(3), 120-128 (2010).

15. Perloff ES, Mason AK, Dehal SS et al. Validation of cytochrome P450 time-dependent inhibition assays: a two-time point IC 50 shift approach facilitates kinact assay design. Xenobiotica 39(2), 99-112 (2009).

16. Venkatakrishnan K, Obach RS. Drug-drug interactions via mechanism-based cytochrome $\mathrm{P} 450$ inactivation: points to consider for risk assessment from in vitro data and clinical pharmacologic evaluation. Curr. Drug Metab. 8(5), 449-462 (2007).

17. US FDA. Guidance for Industry. Bioanalytical Method Validation(2013). www.fda.gov/downloads/drugs/guidances/ucm368107.pdf 
18. Yao M, Zhu M, Sinz MW et al. Development and full validation of six inhibition assays for five major cytochrome P450 enzymes in human liver microsomes using an automated 96-well microplate incubation format and LC-MS/MS analysis. J. Pharm. Biomed. Anal. 44(1), 211-223 (2007).

19. Wu X, Wang J, Tan L et al. In vitro ADME profiling using high-throughput RapidFire mass spectrometry: cytochrome p450 inhibition and metabolic stability assays. J. Biomol. Screen. 17(6), 761-772, (2012).

20. Cece-Esencan EN, Fontaine F, Plasencia G et al. Software-aided cytochrome P450 reaction phenotyping and kinetic analysis in early drug discovery. Rapid Commun. Mass Spectrom. 30(2), 301-310 (2016). 
